\title{
Molecular electronics: history and fundamentals
}

\author{
Santiago Marqués-González, ${ }^{\mathrm{a}, \mathrm{b}}$ Paul J. Low ${ }^{\mathrm{a}, \mathrm{c}}$ \\ ${ }^{a}$ Department of Chemistry, Durham University, South Rd, Durham, DH1 3LE, UK \\ ${ }^{b}$ Department of Chemistry, Graduate School of Science and Engineering, Tokyo \\ Institute of Technology, Meguro, Tokyo 152-8551, Japan \\ ${ }^{c}$ School of Chemistry and Biochemistry, University of Western Australia, 35 Stirling \\ Highway, Crawley, 6009, WA, Australia.
}

\section{Abstract}

The increasing difficulties of meeting “Moore’s Law” rates of progress in conventional semiconductor electronics, coupled with the advent of methods capable of measuring the electronic properties of single molecules in a laboratory setting, has seen a surge of activity in the field of molecular electronics over the last decade. However, the concepts of molecular electronics are far from new, and the basic premise and ideas of molecular electronics have been shadowing those of solid state semiconductor electronics since the middle of the $20^{\text {th }}$ Century. In this Primer Review we introduce the topic of molecular electronics, drawing on some of the earliest expressions of the fundamental concepts, and summarising key concepts to provide the interested reader with an entry to this fascinating field of science and emerging technology. 


\section{Introduction}

Molecular electronics is an interdisciplinary field that involves the use of molecules as fundamental electronic components such as wires, transistors, memory cells, and logic elements. The field, sometimes called "moletronics", [1] is currently attracting attention from a broad cross-section of the scientific community in response to both fascination with the fundamental scientific challenges associated with the measurement of the electrical properties of molecules, and manipulation of electronic phenomena via molecular processes, ${ }^{[2]}$ and growing concerns over the technological challenges and ultimate limits facing solid state semiconductor technology. ${ }^{[3]}$

Before entering discussion, a distinction needs to be made between molecular materials for electronics and single molecule electronics. The use of organic materials for electronic applications in which the bulk electronic or optoelectronic response arises from ensembles of several millions of molecules, and for which properties are measured or observed on the macroscopic level, is at a mature stage of development and application. The most obvious examples of molecular materials readily available in the electronics mass market are the use of liquid crystals and organic light emitting diodes (OLEDs) in flat video displays. ${ }^{[4]}$ This sector of the electronics industry continues its steady development driven by the promises of transparent, flexible, non-toxic, printable electronics, with improved consumer appeal and reduced fabrication costs.

However, the field of single molecule electronics, which is the subject of this review, can be summarised as the use of individual molecules to mimic functional elements in electronic devices. The use of molecules as building blocks to give rise to a more complex system is typically referred to as a the "bottom-up” approach, as opposed to the conventional "top-down" lithographic techniques that are employed to etch small 
features into single-crystal silicon wafers. Despite, or perhaps because of, the great challenges the field of single molecule electronics presents, the enormous benefits of a molecule-based "bottom-up” approach motivates the scientific community to keep moving the field forward. For example, a typical computer microchip nowadays contains of the order of $10^{9}$ transistors in areas of about $3 \mathrm{~cm}^{2}$ with all its components working in perfect harmony and capable of changing state in response to an input signal at an astonishing rate and for an almost unimaginable number of cycles. The enormous challenge for single molecule electronics lies in the development of molecular systems that perform in some way better than, or perhaps provide an alternative function to, these extraordinary solid-state devices.

\section{Molecular and semi-conductor electronics: a brief account of an interwoven}

\section{history}

To comprehend the incentives that drive the development of molecular electronics (Figure 1, right), it is necessary to first appreciate the evolution of the silicon microelectronics industry (Figure 1, left). ${ }^{[5]}$ The first experiments concerning the electrical properties of semiconductor materials began early in the $19^{\text {th }}$ century. The surprising properties of these materials, such as their increased conductance when heated or exposed to light, captured the attention of scientists and engineers of the time. Amongst the most renowned contributors of the time are Michael Faraday, who first reported the conductance increase with temperature in silver sulfide (1833), AlexandreEdmond Bequerel, father of the photovoltaic effect (1839), and Alexander Graham Bell who in 1880 took advantage of photo-sensitivity of selenium to transmit sound over a beam of light, inadvertently heralding the fiber-optic communications revolution of the 
$21^{\text {st }}$ Century, with a remarkable invention that he named the "photophone" (US235199A).

In spite of the potential of these early experiments, it is the development of the solid-state transistor that might best be considered to mark the beginning of the semiconductor revolution. Shortly after the end of World War II (1947) Walter Brattain, John Bardeen and William Shockley of Bell laboratories discovered that by applying a small bias to the surface of a germanium block, the current flow through a second circuit connected to that piece of germanium could be modulated, thereby providing the first demonstration of a solid-state transistor. 


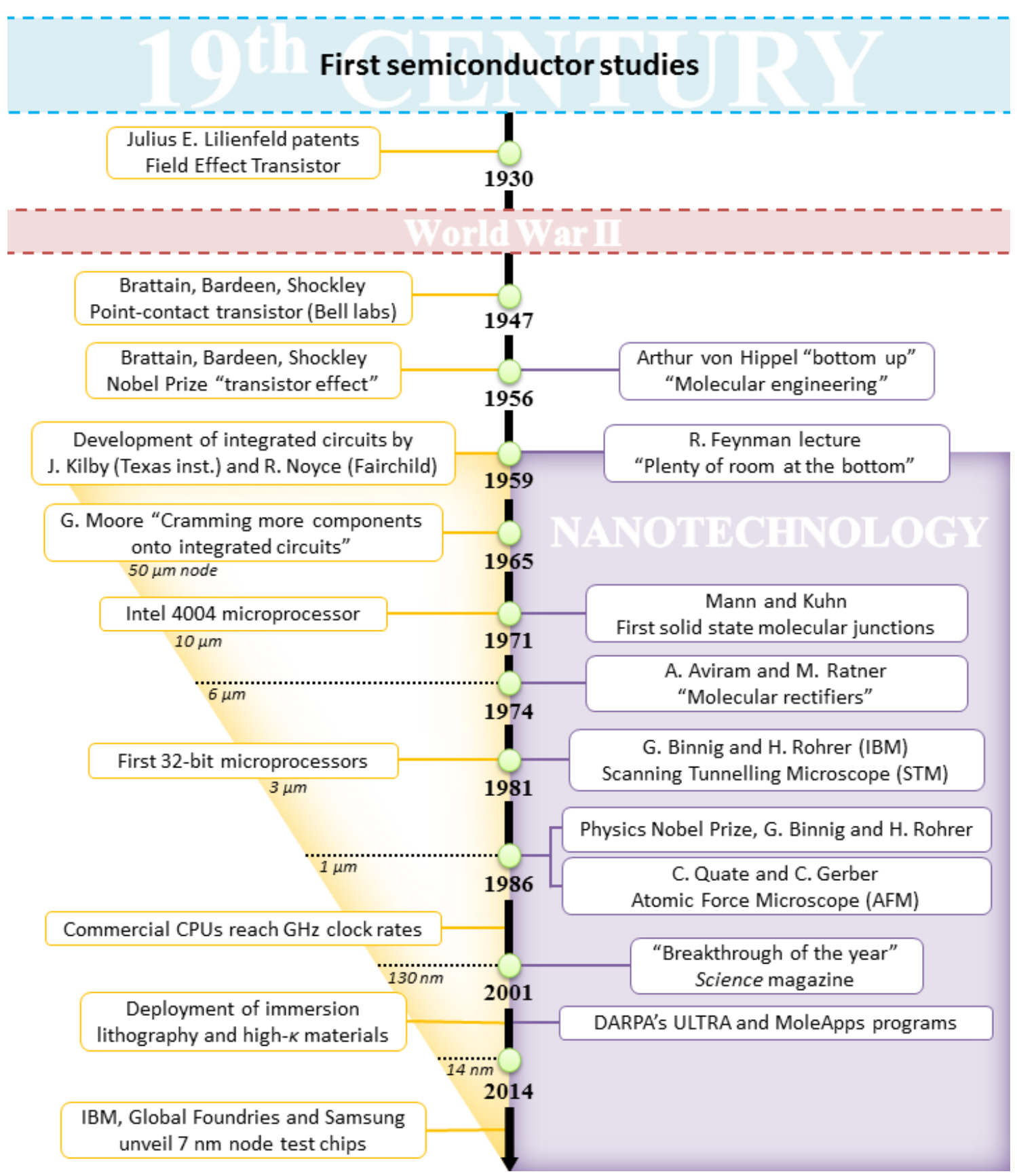

Figure 1. Silicon industry and molecular electronics roadmap, from the genesis of the

first solid-state transistor to our days.

However, in an often overlooked episode of the semiconductor history, half of Bell laboratories initial patent claims in the area were rejected in benefit of Julius E. Lilienfeld, an Austro-Hungarian physicist best known for the discovery of radiation emitted by a metallic surface when electrons strike it, a process now known as 
Lilienfeld radiation. In 1930, seventeen years before the Bell labs discovery, Lilienfeld patented the 'field-effect transistor' (US1745175A). Despite his early description of the transistor effect, Lilienfeld's work was largely ignored by the emerging semiconductor industry. Bell patents were finally issued for the "point-contact transistor" by Brattain and Bardeen (US2524035A) and the "junction transistor" by Shockley (US2569347A). In 1956, only nine years after the first transistor was built, Brattain, Bardeen and Shockley were awarded the Physics Nobel Prize for the discovery of the transistor effect. In recognition of Lilienfeld's sometimes overlooked contribution to science, an annual prize that carries his name was established in 1988 by the American Physical Society to reward a most outstanding contribution to physics.

The 1950s was an era of frenetic activity at the Bell laboratories aligned around the production of solid-state electronics. Parallel to the fabrication of the first transistors, Jules Andrus and Walter L. Bond began adapting a photoengraving technique used to print patterns onto integrated circuits to generate sophisticated designs on silicon wafers. The solid-state transistor and photolithographic process, together with the serendipitous discovery in 1955 of silicon oxide masking by Carl Frosch, also at Bell, marked the start point for the modern monolithic microelectronics industry. However, the same year that the Physics Nobel Prize was awarded for the discovery of the transistor effect (1956), a German physicist named Arthur von Hippel, then working at the Massachusetts Institute of Technology, proposed a completely different approach:

"Instead of taking prefabricated materials and trying to devise engineering applications consistent with their macroscopic properties, one builds materials from their atoms and molecules for the purpose at hand ... 
The notion of "molecular engineering" introduced by von Hippel might be the first expression of the "bottom-up" approach and could be regarded as the initial expression of the concept of molecular electronics. von Hippels's ideas were quickly embraced by the Westinghouse Electric company, ${ }^{[7]}$ and the US Air Force (USAF). At that time, the airborne electronic equipment was growing increasingly complex and vulnerable to failure, as aircraft began flying faster and higher. In 1959 the USAF decided to invest US\$2 million in a joint USAF-Westinghouse research program for the development of molecular electronics as a possible solution to their problems. Their research proposal “Molecular Electronics - Dendritic Approach” proved successful enough to be funded until 1962, although the research program was ultimately abandoned due to severe manufacturing issues and the rapid progress of the silicon electronics technology. Coincidently, also in 1959 Jack Kilby at Texas Instruments, and Robert Noyce at Fairchild Camera, had developed a method to integrate even more of the individual components to create a 'solid circuit'. The ability to print and wire several electronic components on a silicon substrate, coupled with reduction in the size of components promised to reduced production costs and increase reliability. The importance of their work was recognized later in 2000 when Kilby was awarded the Physics Nobel Prize for his contribution in the invention of the integrated circuit.

The desire for smaller, faster, more highly functional electronic components gave rise to the rapid post-war evolution of the silicon industry, led by development of the integrated circuit and economic drivers. In 1965, a marketing research paper “Cramming more components onto integrated circuits” was published by Gordon E. Moore, then at Fairchild Semiconductors. ${ }^{[8]}$ In this study, Moore noted the doubling of component density every two years on electronic circuits, and projected that such rates of progress might be possible until 1975 (!). Only three years later, in 1968, Moore, 
together with Noyce, founded the Intel Corporation, and in 1971, the Intel 4004 processor was introduced, with over 2000 transistors built on a silicon monolith the size of a fingernail. The journey towards the evolution of consumer electronics and the multi-billion dollar semiconductor industry had begun. As semiconductor fabrication techniques evolved, Moore’s expression of component density doubling every two years became a self-imposed growth target for the semiconductor industry. The desire to maintain this rate of progress, typically referred to as "Moore's Law", has driven the relentless miniaturization efforts of the semiconductor industry for five decades.

In contrast, the ideas of molecular electronics technology laid largely fallow until the 1970s, when a second phase of interest swept the community. Again in 1959, not long before Moore's seminal expression of the rates of progress in the semiconductor industry based on economic considerations, Richard Feynman gave his now widely celebrated lecture to the American Physical Society at CalTech titled "There's plenty of room at the bottom" in which he challenged the whole scientific community to push the miniaturization limits. ${ }^{[9]}$ For many, the ideas Feynman exposed in that lecture are the starting point of nanoscience and nanotechnology. In a remarkable piece of foresight and imagination Feynman succinctly drew together the threads of both the fast emerging microelectronics industry with a future molecular electronic technology by noting that, en route to an ultimate miniaturisation of technology, chemical synthesis allows for the precise organisation of matter within a molecular framework.

At that time, although chemical synthesis was (relatively) well developed, establishing electrical contacts across individual molecules was not possible; however intramolecular electron transfer was being increasingly studied in solution, with considerable interest in using the characteristic spectroscopic profiles associated with 
'mixed valence' complexes and compounds, led by the seminal work of Taube, Marcus and Hush. ${ }^{[10]}$ Indeed, the use of mixed-valence systems as models for molecular electronics components continues to this day, although the neglect of the surfacemolecule interface in these models is noted. ${ }^{[11]}$

The first direct measurements of through molecule conductance were reported in 1971 when Mann and Kuhn ${ }^{[12]}$ prepared a series of well-ordered fatty acid monolayers employing the Langmuir-Blodgett (LB) technique, and succeeded in sandwiching them between metal electrodes and measuring the electrical properties of the systems. Their studies revealed an exponential decay of the conductivity with the molecular length. Molecular electronic concepts began to take further formative and conceptual steps through the 1970s. In 1974, Ari Aviram (IBM) and Mark Ratner (New York University) started working on a theory of electron transfer through single organic molecules. Their efforts crystallized in a now-famous article titled "Molecular Rectifiers". ${ }^{13]}$ In that document, Aviram and Ratner described for the first time the use of a molecule with a modular design based on fragments familiar to chemists as an electronic component (Chart 1).

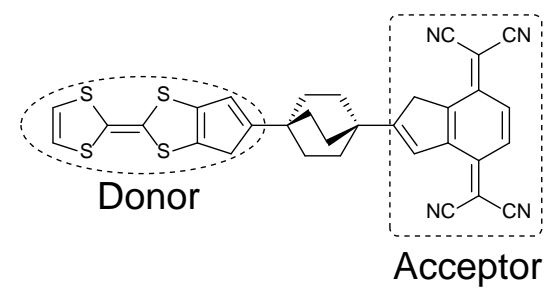

Chart 1. The Aviram and Ratner molecular rectifier based on tetrathiafulvalene (donor) and tetracyanoquinodimethane (acceptor) linked via a saturated methylene bridge.

Curiously, Aviram and Ratner never used the words "molecular electronics" in their article and, to the best of our knowledge, this compound has not yet been 
synthesized; nevertheless, their seminal work became a cornerstone of contemporary molecular electronics with over 2000 citations and a vast body of work on unimolecular rectifiers through the 1980 s.

The invention and development of the scanning tunnelling microscope (STM) by Gerd Binnig and Heinrich Rohrer at IBM in 1981 (for which they were awarded the 1986 Nobel Prize in Physics) and the atomic force microscope (AFM) later that year by Calvin Quate and Christoph Gerber provided further stepping stones to the development of molecular electronics. With the introduction of the STM and the AFM, the experimental barrier which had frustrated every previous attempt to directly evaluate the electronic properties an individual molecule was largely overcome. The maturing of the STM, and the development of a number of scanning probe microscopies, ${ }^{[14]}$ such as the conducting probe atomic force microscopy (CP-AFM), together with the rise of interest in nanotechnology ${ }^{[15]}$ brought a second renaissance in molecular electronics during the 1990s.

In an echo of the early USAF-Westinghouse project, molecular electronics captured the attention of the Defence Advanced Research Projects Agency (DARPA) and an initial proposal by Mark Reed and James Tour to the Office of Naval Research, titled “Spontaneously-assembled molecular transistors and circuit”, became DARPA's ULTRA (for ultrafast, ultradense electronics) research theme. DARPA consolidated the ULTRA program as a Moletronics research program to provide funding to a multidisciplinary research network from the year 2000, which was overtaken by the Applications of Molecular Electronics (MoleApps) programme in 2004. During this period, a considerable body of work with carbon nanotube based devices and fundamental work concerning the measurement of single molecule conductance was undertaken, as can be seen in the growing number of research articles in these areas 
published during the second half of the 1990s. A series of quite remarkable achievements in 2001 including the creation of carbon nanotube based transistors, ${ }^{[16]}$ switchable devices from rotaxanes embedded between $n$-type polysilicon and Ti/Al electrodes $^{[17]}$ and reports of 16-bit memory from similar rotaxanes in cross-bar junctions lead the Science magazine to describe 'Nanoelectronics' and the construction of these rudimentary circuits the breakthrough of the year in $2001 .^{[18]}$ In recent years, these concepts have evolved and devices as complex as 160-kb rotaxane-based crossbar memories have been described. ${ }^{[19]}$

Against this background of steady progression in molecular electronics, the semiconductor industry has pushed relentlessly forward at Moore's Law pace until the present day. ${ }^{[20]}$ The miniaturization race required remarkable innovations in microarchitecture designs such as 3D transistors (tri-gate or FinFET), ${ }^{[21]}$ technical developments such as immersion lithography and double patterning, and the use of exotic high- $\kappa$ dielectrics to alleviate tunnelling issues of gate-insulators of only a few atoms thick. ${ }^{[22]}$ However, the difficulties involved in maintaining Moore's Law rates of miniaturization below the $20 \mathrm{~nm}$ barrier is perhaps best illustrated by the delayed deployment of the $14 \mathrm{~nm}$ chips (Intel Broadwell, 2015). Whilst a miss-step in Intel's production schedule and failure to meet its long standing 12-18-month 'tick-tock' schedule of chip refresh (tick $=$ shrinking of the process technology; tock $=$ new microarchitecture), may not seem a major economic or technological concern, this missed production window is an indication of the growing difficulties that conventional semiconductor fabrication methods are now facing. In fact, the challenges associated with manufacturing advanced electronic circuits are now so great that in the international community has been forced to re-write Moore’s Law: 


$$
\text { years. }{ }^{[23]}
$$

Nevertheless, the miniaturization race continues, as it was recently proven by IBM, Global Foundries and Samsung research alliance, with the development of the first $7 \mathrm{~nm}$ node test chips at the SUNY Polytechnic Institute's College of Nanoscale Science and Engineering. This astonishing feat required several industry-first innovations such as the fabrication of Si-Ge transistor channels, extreme ultraviolet lithography, and allegedly quadruple patterning.

As the semiconductor industry approaches the physical limits of solid-state materials, molecular electronics is entering something of a third era, this time supported by shared academic and industrial interest, with an appreciation of the need to solve fundamental problems in order to reach a hybrid electronics technology in which molecular systems serve as a viable material within solid state frameworks. However, the complex experimental set-up required to determine the electrical properties of a single molecule has historically been, and to some extent still is, one of the most important obstacles to the exploration of fundamental concepts in molecular electronics and advancement towards a viable technology. Nevertheless, the invention of the STM and AFM and improvements in nanofabrication, now present us with a number of methods that allow the formation of molecular junctions in order to study their physical properties and electronic performance have been firmly established. ${ }^{[24]}$ The main aim of these methods is to assemble either one or a small number of molecules between two metallic electrodes to create a junction, which in turn allows the electronic properties of the sandwiched molecules to be measured. ${ }^{[25]}$ In the remainder of this article we turn attention to these molecular junctions, and explore the basic processes that underpin the electrical properties of molecules within electrode gaps. 


\section{Molecular junctions}

A molecular junction consists of a molecule suspended or sandwiched between two (usually metallic) electrodes. When a molecule is brought in contact with a metallic electrode, the molecular orbitals and the electrode states overlap to a certain extent to form a new hybrid electronic wavefunction. The degree of coupling may vary from conjugated states extending over the whole molecular junction, to the generation of orbital nodes along the junction acting as barriers to electronic transport between the electrodes. Despite the great progress made in this field, and the apparent simplicity of the sandwich style molecular junction (Figure 2), a full theoretical description of metal|molecule|metal junctions and a detailed understanding of structure-property relationships, remain as challenges.

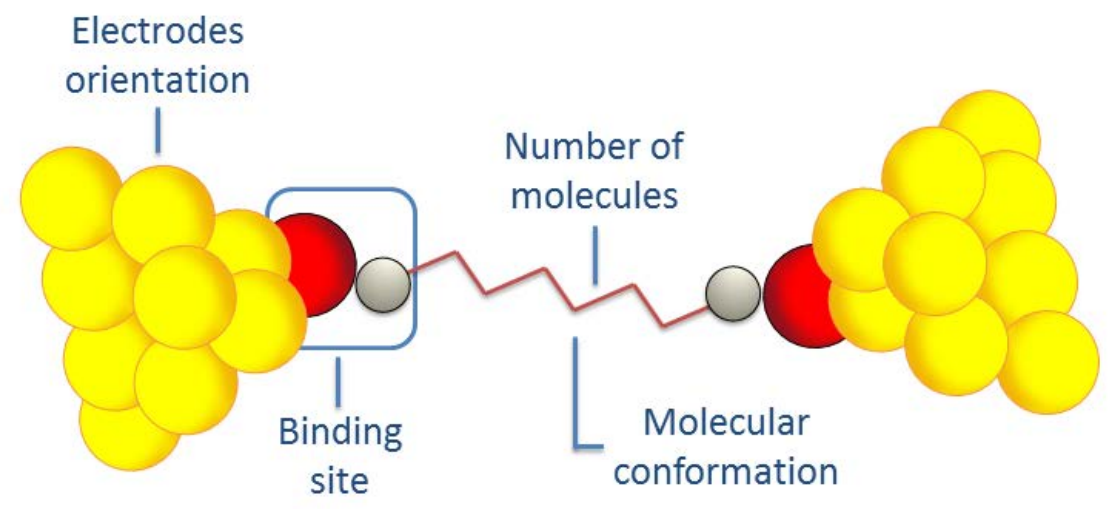

Figure 2. Schematic representation of a fully characterized molecular junction where: binding sites; electrodes orientation; molecular conformation and number of molecules are accurately characterized.

On the basis of the plethora of studies on molecular junctions that have been reported over the past decade, it has been noted that the conductance of a molecular 
junction can be affected by several factors such as: the structure and degree of conjugation of the molecular bridge, ${ }^{[26]}$ the nature of the linker group and its geometry; ${ }^{[27]}$ the junction geometry (tilt angles and gap size) ${ }^{[28]}$ and the electronic character of substituents on the molecular backbone. ${ }^{[29]}$

Much of the early work in single molecule studies was focused on the molecular backbone assuming that any other factor would have a small influence in the junction electronic performance. Particularly large contributions to this field have come from studies of symmetrically and unsymmetrically substituted linear oligo(phenyleneethynylene) (OPE) derivatives, ${ }^{[30]}$ including organometallic analogues. ${ }^{[31]}$ These highly conjugated derivatives, sometimes referred to as "Tour wires" due to the pioneering studies of these molecules by J. M. Tour, ${ }^{[32]}$ have been of significant interest in many different fields of chemistry and physics due to their exceptional properties. ${ }^{[33]}$ The modular synthesis of OPEs, ${ }^{[34]}$ and good single molecule conductance ensure that these systems merit particular attention. ${ }^{[26 \mathrm{~b}, 35]}$

However, despite the great interest in the design of conjugated wire-like backbones for use in molecular electronic components, it is now agreed that the metalmolecule interface, and therefore the molecular anchoring group, have a great influence on the global junction behaviour. ${ }^{[27 a, 27 b]}$ This area of research has been largely dominated by the thiolate linker that, due to its ability to self-assemble on gold has provided a strong test-bed to study the electrical properties of single molecules and ensembles. ${ }^{[36]}$ However, several potential disadvantages have been reported to the use of thiols as molecular linkers. The thiolate-gold bond has a strength similar to that of the gold-gold bond resulting on the electrode surface modification. ${ }^{[37]}$ In addition, at room temperature, stochastic switching of the junction conductance has been observed, ascribed to the mobility of the chemisorbed contacts. ${ }^{[38]}$ The free thiols also tend to 
oxidise to give dithiolates, often requiring the use of protective groups during their synthesis. Due to these difficulties and the influence of the linker on the junction properties, the development of alternative contacts has become an area of great research activity and several alternative linkers to thiols have been proposed. ${ }^{\text {[27c-f, 39] }}$

In addition to the linker, the exact separation between the two electrodes when the molecular bridge is formed can have a pronounced influence in the junction conductance. This is extremely relevant to the development of molecular electronic devices that can show a considerable range of contact-gap separation. Haiss et al. demonstrated that the molecular conductance can be measured as a function of electrode gap to sub-nanometre precision. ${ }^{[28]}$ More recently, the effect of the junction gap was also studied for a series of rigid molecular wires. ${ }^{[28 b]}$ In those studies, a substantial conductance increase was found as the gap between the electrodes was closed. DFT studies supported those results proving the significant influence of the molecular tilt angle in the junction conductance. ${ }^{[28 a]}$

A simplified electronic description of a molecular junction involving two electrodes bridged by a molecule can be seen in Figure 3. Both electrodes are described as a continuum of energy levels filled up to a given energy level (Fermi level), the energy symmetry between both electrodes is broken by the applied bias. On the other hand, the bridging molecule is characterized by discrete energy levels filled up to the HOMO. Importantly, the orbital alignment relative to the electrode Fermi level is directly characteristic of each molecular junction and dependant on several factors such as: the nature of the molecular bridge; ${ }^{[40]}$ the nature of the metal-molecule interaction; ${ }^{[27 e]}$ the electronic and conformational changes induced by the charge transfer; ${ }^{[41]}$ environmental effects ${ }^{[25 b]}$ and redox state. ${ }^{[25 b, 42]}$ 


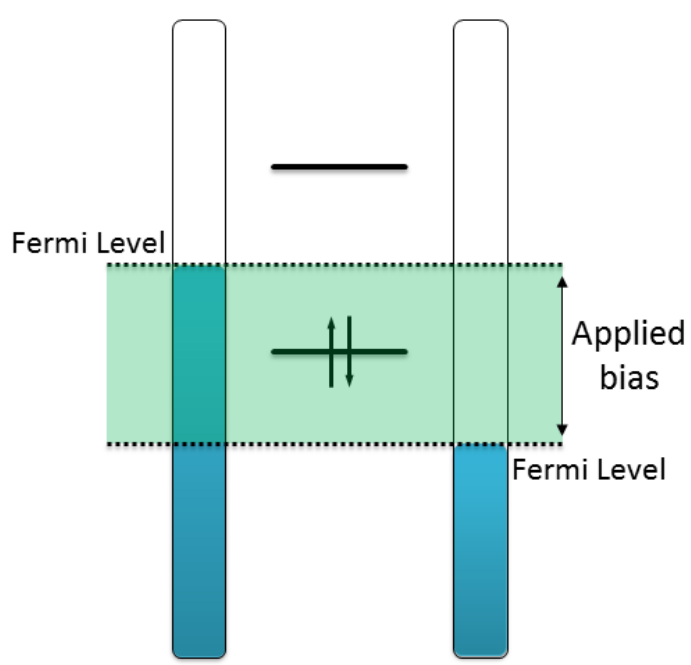

Figure 3. Simplified description of the electronic diagram in a molecular junction.

Charge transport in molecules contacted by macroscopic electrodes is best described by the Landauer formalism. In this description electrons are treated as waves that can be reflected or transmitted through the molecular bridge. According to the Landauer formalism the conductance $G$ of a molecular junction can be calculated (Equation 1).

$$
G=\frac{2 e^{2}}{h} \sum_{n} T_{n} \quad \text { Equation } 1
$$

where $e$ is the electron charge, $h$ is Planck's and $T_{\mathrm{n}}$ are the transmission coefficients of the individual transport channels. According to this expression, the conductance of a system $G$, is the summation of all possible individual transmission channels. Perhaps the most relevant implication of this equation is that conductance at the molecular level is quantized. For a perfect coupling i.e. ballistic conductors $\left(T_{\mathrm{n}}=1\right)$, the conductance can only increase or decrease in units of the quantum of conductance $G_{0}=2 e^{2} / h \sim$ $77481 \mathrm{nS}$. It is important to clarify that the Landauer formalism does not imply that the conductance of any system must be an integer of $G_{0}$ however it defines the maximum 
conductance for a single channel as the $G_{0}$. Transmission coefficients $T_{\mathrm{n}}$ typically take values smaller than the unity and several transport channels coexist to give the system conductance. Conductance values for single molecules are orders of magnitude smaller than $G_{0}$ typically ranging from $\left(10^{-5}-10^{-1}\right) G_{0}$.

Several theoretical models have been developed to describe the electron transport through molecular junctions, the temperature independent coherent tunnelling (superexchange) and Fowler-Nordheim tunnelling; and the temperature dependent thermionic (Schottky) emission and hopping conduction. ${ }^{[43]}$ Despite the multiple models developed for temperature dependent and independent processes (mainly differing on their bias dependence), for simplicity here we will only differentiate between tunnelling (temperature independent) and hopping (temperature dependent) processes. A more complete description and discussion on this topic is available in several reviews. ${ }^{[44]}$ Although, typically only one of these mechanisms dominates the charge transfer through the molecular junction, both tunnelling and hopping mechanisms can coexist. ${ }^{[45]}$ Hence, the observed rate of electron transfer $\left(k_{\mathrm{et}}\right)$ results from the summation of the tunnelling $\left(k_{\mathrm{tunn}}\right)$ and hopping $\left(k_{\mathrm{hop}}\right)$ contributions. A schematic representation of both charge transport processes is shown in Figure 4.
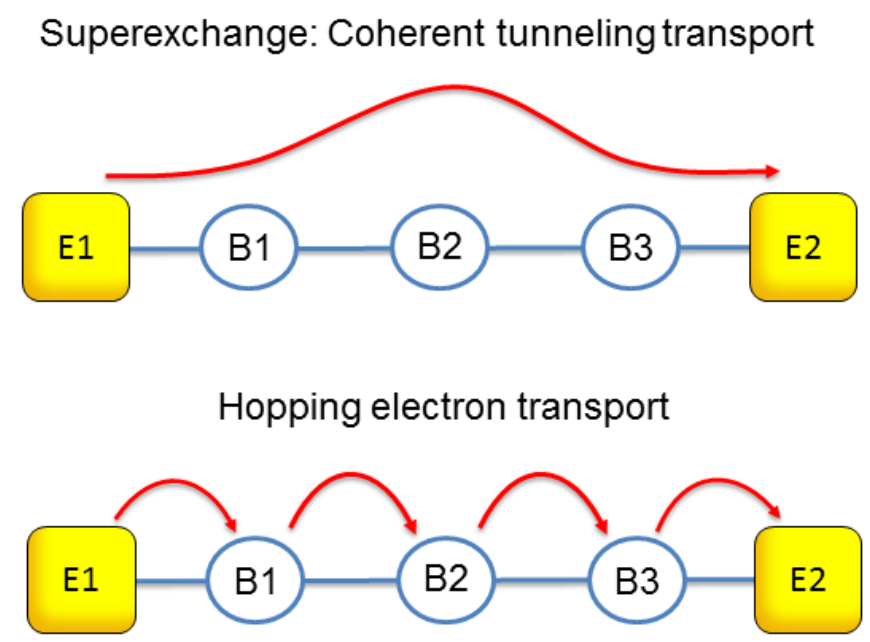

Figure 4. Schematic representation of the superexchange and hopping mechanisms. 
The super-exchange mechanism is based on the probability of an electron traversing an energy barrier coherently i.e in the absence of inelastic scattering events. In this case, the charge transfer takes place in one step through the molecular orbitals of the bridging molecule. Hence, the charge carrier is considered not to reside in the orbitals of the bridging molecular wire for a significant space of time. It is important to note that, the electron transference $k_{\mathrm{ET}}$ is enhanced by the molecular orbitals increasing the probability of through-bond tunnelling over trough-space tunnelling. In this scenario the energy barrier is set by the energetically higher electronic states of the bridge compared to those of the electron source. The electron transport rate $k_{\mathrm{ET}}$ for the superexchange mechanism can be calculated using Equation 2

$$
k_{E T}=k_{0} e^{-\beta r} \quad \text { Equation } 2
$$

where $k_{0}$ is a kinetic prefactor, $r$ is the distance between the two electrodes and $\beta\left(\mathrm{nm}^{-1}\right)$ is the tunnelling attenuation factor. As it derives from Eq. 2 the super-exchange mechanism is temperature independent and exponentially dependent on the electrode distance $r$. The attenuation factor $\beta$, represents the degree of electronic coupling present along the molecular junction and enables direct comparison between single molecule studies. Typically the decay constant $\beta$ for $\pi$-conjugated molecules is an order of magnitude smaller $\left(1-2 \mathrm{~nm}^{-1}\right)$ than that of the saturated $\sigma$-bonded chains $c a .10 \mathrm{~nm}^{-1}{ }^{[40}$, 46] Several distance dependence studies have reported $\beta$ values for a number of molecular systems: $3.4 \mathrm{~nm}^{-1}$ for di-thiol terminated OPEs; ${ }^{[47]} 2.0 \mathrm{~nm}^{-1}$ for amine terminated OPEs; ${ }^{[35 e]} 3.3 \mathrm{~nm}^{-1}$ for pyridine terminated OPEs; ${ }^{[39 \mathrm{e}]} 3.5-5 \mathrm{~nm}^{-1}$ for di-thiol terminated oligophenylenes; ${ }^{[48]} 1.8 \mathrm{~nm}^{-1}$ for oligophenylene-vynylenes; ${ }^{[49]} 1 \mathrm{~nm}^{-1}$ for dithiocyanate terminated oligothiophenes; ${ }^{[46]} 0.6 \mathrm{~nm}^{-1}$ for pyridine terminated 
oligoynes; ${ }^{[39 b]}$ as opposed to $9.4 \mathrm{~nm}^{-1}$ for saturated di-thiol terminated alkyls ${ }^{[48 \mathrm{a}]}$ and the $24-40 \mathrm{~nm}^{-1}$ for vacuum. ${ }^{[50]}$ It is important to note that, the attenuation factor $\beta$ not only depends on the orbital delocalization of the molecular backbone but on the entire molecular junction, including the binding sites and the electrode shape and material (i.e. the measuring technique). ${ }^{[49 a]}$ However, due to the exponential distance dependence of the superexchange mechanism, coherent tunnelling is only effective for distances under $2.5 \mathrm{~nm}$. The charge hopping mechanism is believed to dominate for longer bridges (ca. $3 \mathrm{~nm})$

Contrary to the super-exchange mechanism, in the case of charge hopping, the electron traversing the molecular wire is localized for a short time before moving to the next bridge site until it crosses trough the molecular junction. ${ }^{[49 \mathrm{~b}, 51]}$ The hopping conduction mechanism is more likely to be present in molecular junctions where the Fermi levels of the metallic contacts lie close in energy to the molecular bridge frontier orbitals. ${ }^{[43]}$ Importantly, significant nuclear motion is involved in this transport process as vibrational relaxation takes place at each bridge site. Consequently, the distance dependence of charge hopping is less marked, i.e. inversely proportional to distance (Ohmic) (Equation 3) (although other relationships are also known (e.g. $k_{E T}=k_{h o p} N^{2}$, where $N$ is the number of hopping stations in the bridge)). ${ }^{[45]}$ In addition, due to the vibrational relaxation processes involved the hopping mechanism is temperature dependant. $^{[43,45]}$

$$
k_{E T}=k_{\text {hop }} \propto 1 / r \quad \text { Equation } 3
$$

In order to experimentally determine the transport process ruling in a given molecular junction, two electrical measurements are typically performed: the length dependence of the molecular conductance and the temperature dependence of the $I-V$ 
profile. These experiments also exclude the possibility of artefacts such as metallic filaments or interface effects being the source of the electrical properties registered. However, the determination of the charge transport mechanism can sometimes be difficult. For example, a pronounced temperature dependence was reported for the conductance of alkanedithiols attributed to changes in the distribution of the molecular conformers.

The coexistence of both tunnelling and hopping mechanisms was first reported by Wasielewski and Ratner. ${ }^{[52]}$ In their work, the recombination rate of several photoexcited Donor-Bridge-Acceptor (D-B-A) complexes featuring p-phenylenevinylene oligomeric bridges $(n=1-5)$ was studied. Notably, a less marked distance dependence was found for those molecular bridges with sizes over $2.4 \mathrm{~nm}$ that was attributed to a change in the transport mechanism from superexchange ( $n=1-2)$ to hopping ( $\mathrm{n}=3$ - 5). Choi and co-workers reported a similar behaviour for phenyleneimine oligomers $(n=1-10)$ in solid state CP-AFM studies. ${ }^{[53]}$ Those results confirmed the coexistence of both transport mechanisms in the solid state. In this case, transport mechanism was reported to change from tunnelling to hopping for molecular wires over $4 \mathrm{~nm}$ in size $(n=6-10)$. Despite the great experimental complexity of these experiments, a number of similar studies have been reported recently confirming the coexistence of both mechanisms. ${ }^{[35 e, 39 e, 54]}$ Although in first instance the charge hopping mechanism and its weaker distance dependence should allow long distance charge transport, which makes it more attractive for potential electronic application, the tunnelling mechanism is still intriguing the scientific community. For instance, recent studies indicate that tunnelling-energy gap effects can differentiate the distance dependence of energy-storing charge-separation reactions from energy-wasting 
recombination processes. ${ }^{[50 \mathrm{~b}]}$ Hence further understanding of the tunnelling mechanism may provide an additional way to obtain long-living charge-separated states.

\subsection{Relationships between electron transfer rates and molecular conductance.}

As the number of single molecule studies increases, several theoretical studies have investigated the relation between the electron transfer rates $\left(k_{\mathrm{DA}}\right)$ obtained from the in-solution studies and the molecular conductance $(G)$ obtained for molecular junctions. ${ }^{[55]}$ Despite the fundamental differences behind the two physical systems both processes depend on quantum tunnelling to carry the charge through the molecular bridge. ${ }^{[4 \mathrm{~b}, 56]}$ Hence, the conduction profile of a given system and its electron transfer properties must be closely related. ${ }^{[55 a]}$ It is important to bear in mind that, because of the tunnelling regime there is always an Ohmic behaviour region near zero bias. On the basis of this similarity first noted by Nitzan, ${ }^{[55 a, 57]}$ the relation between the rate constant $k_{\mathrm{AD}}$ and conductance $G$ is shown in Equation 4

$$
G \approx \frac{e^{2}}{\Gamma_{D} \Gamma_{A} F} k_{D A} \quad \text { Equation } 4
$$

where $e$ is the electron charge, and the $\Gamma$ factors are the inverse lifetimes of an electron on the donor and acceptor states once the molecular junction is formed, and $F$ is the Marcus thermally averaged Franck-Condon factor dependant of reorganization energy and temperature. However this relation is only applicable for the simple case of thermal, non-adiabatic electron transfer in those junctions where the molecular electronic structure is not greatly affected by the metal-molecule interaction. A rough estimation of this relation for a junction with typical magnitudes of reorganization energy and

metal-molecule coupling was done by Nitzan leading to $G(\mathrm{~S}) \sim 10^{-17} k_{\mathrm{DA}}\left(\mathrm{s}^{-1}\right) .{ }^{[55 a]}$ Despite the fact that this theoretical model was first developed for the tunnelling 
mechanism, it was later extended to the hopping mechanism. ${ }^{[57]}$ For those cases where the charge transfer takes place through a large number of bridge sites the relation between $G$ and $k_{\mathrm{DA}}$ was found to be

$$
G \approx \frac{e^{2}}{k_{B} T} e^{-\frac{\Delta E}{k_{B} T}} k_{D A} \quad \text { Equation } 5
$$

where $k_{\mathrm{B}}$ is the Boltzmann constant, $e$ is the electron charge and $\Delta E$ is the difference between the activation energies involved. Interestingly at $T=300 \mathrm{~K}$, for those cases where $\Delta E \leq k_{B} T$, Eq $1-14$ can be simplified as $G(\mathrm{~S}) \sim\left(5 \cdot 10^{-18}\right) k_{\mathrm{DA}}\left(\mathrm{s}^{-1}\right)$ remarkably similar to the numerical estimate obtained for the coherent tunnelling mechanism.

Recently, Wierzbinski and co-workers reported the first experimental study of this relationship. ${ }^{[54 b]}$ In their work, they evaluated the correlation between molecular conductance and electrochemical rate constants for alkanes and nucleic acid oligomers as a function of length, structure and charge transport. Interestingly, contrary to the linear correlation theoretically proposed by Nitzan a power law correlation was found between $G$ and $k_{\mathrm{DA}}$ for each molecular bridge studied. The deviation from the linear relation was attributed to charge-transfer energy barriers and bridge dephasing. In situations where multiple mechanisms can coexist, these factors can lead to differences between the distance dependence of $k_{\mathrm{DA}}$ and $G$. Generally speaking, these results show the relative propensity of different chemical species to transmit charge differently in electrochemical measurements as opposed to molecular junctions.

\section{Conclusion and future prospects in molecular electronics}

Since its inception some 60 years ago the field of molecular electronics has surged, ebbed, and surged with conceptual, technical and scientific advances. The great 
advances in our understanding of the unique and complex nature of charge transport at the molecular level, together with a growing and increasingly recognised industrial need, may, perhaps finally, see the potential of molecular electronics realised.

Through the wider access to the experimental tools that allow single molecule measurements, many new charge transport phenomena beyond the simple ballistic electron transport have been identified. Amongst those phenomena, molecular rectifying (redox, thermal or mechanic), quantum-interference, spintronics and optoelectronics are now being actively pursued, and open avenues for exploration of both organic compounds and metal complexes. In addition, theoretical models and computational methods based on the non-linear Green's function (NLGF) are providing the theoretical base required to fully understand the transport process at the nano-scale. A full description of the theoretical rules that drive the molecular conduction is of great importance as it would lead to molecular behaviour prediction and therefore to a rational design of the molecules.

Molecular electronics seems predestined to converge with the highly developed semiconductor industry, in a curious reunification of ideas shared a common heritage and conceptual beginning. The great effort now being devoted to increase the compatibility of molecular electronics with the fabrication methods employed by the semiconductor industry heralds a new initiative in realising a true molecular electronics technology. ${ }^{[23]}$

Computing with molecules as circuit building blocks is an exciting concept with several desirable advantages over conventional circuit elements. Because of their small size, very dense circuits could be built, and bottom-up self-assembly of molecules in complex structures 
could be applied to augment top-down lithography fabrication techniques. As all molecules of one type are identical, molecular switches should have identical characteristics, thus reducing the problem of variability of components. However, the success of molecular electronics depends on our understanding of the phenomena accompanying molecular switching, where currently many questions remain.

The obstacles to this goal should be seen as challenges for the scientific community, acting as motivation and defining the long-term goals of the field. In the words of Heath and Ratner, ${ }^{[58]}$ on the molecular electronics horizon should be placed a very robust, energy-efficient, connected to the outside world, computational platform based on molecular electronics with a bit density of $10^{12} \mathrm{~cm}^{-2}$. The great advances needed to accomplish that goal, would make it hard to believe that an electronic device will be the most significant result of such an effort.

\subsection{References}

[1] K. S. Kwok, J. C. Ellenbogen, Materials Today 2002, 5, 28.

[2] (a) F. Chen, N. J. Tao, Accounts of Chemical Research 2009, 42, 429; (b) R. M. Metzger, Journal of Materials Chemistry 2008, 18, 4364. 
[3] (a) R. K. Cavin, P. Lugli, V. V. Zhirnov, Proceedings of the IEEE 2012, 100, 1720; (b) A. Huang, Spectrum, IEEE 2015, 52, 43; (c) N. Collaert, A. Alian, H. Arimura, G. Boccardi, G. Eneman, J. Franco, et al., Microelectronic Engineering 2015, 132, 218.

[4] (a) I.-C. Khoo, I. Wiley, Liquid crystals 2007 (Wiley-Interscience: Hoboken, N.J., USA); (b) A. P. Kulkarni, C. J. Tonzola, A. Babel, S. A. Jenekhe, Chemistry of Materials 2004, 16, 4556; (c) H. Sasabe, J. Kido, Chemistry of Materials 2010, 23, 621; (d) C. Zhong, C. Duan, F. Huang, H. Wu, Y. Cao, Chemistry of Materials 2010, 23, 326.

[5] H. Choi, C. C. M. Mody, Social Studies of Science 2009, 39, 11.

[6] A. von Hippel, Science 1956, 123, 315.

[7] E. A. Sack, D. A. Lewis, IEEE Annals of the History of Computing 2012, 34, 74.

[8] G. E. Moore, Electronics Magazine 1965, 38, 8.

[9] (a) 'Plenty of room' revisited [Editorial], Nature Nanotech. 2009, 4, 781; (b) R. P. Feynman, Caltech Engineering and Science 1960, 23:5, 22.

[10] (a) M. B. Robin, Inorganic Chemistry 1962, 1, 337; (b) P. Day, Inorganic Chemistry 1963, 2, 452; (c) D. Culpin, P. Day, P. R. Edwards, R. J. P. Williams, Chemical Communications (London) 1965, 450; (d) C. Creutz, H. Taube, Journal of the American Chemical Society 1969, 91, 3988; (e) G. C. Allen, N. S. Hush. in Progress in Inorganic Chemistry 2007, pp. 357-389 (John Wiley \& Sons, Inc.: ; (f) P. Day, N. S. Hush, R. J. H. Clark, Philosophical Transactions of the Royal Society A: Mathematical, Physical and Engineering Sciences 2008, 366, 5.

[11] J.-P. Launay, Coordination Chemistry Reviews 2013, 257, 1544.

[12] B. Mann, H. Kuhn, Journal of Applied Physics 1971, 42, 4398.

[13] A. Aviram, M. A. Ratner, Chemical Physics Letters 1974, 29, 277.

[14] R. J. Hamers, The Journal of Physical Chemistry 1996, 100, 13103.

[15] Y. Xia, J. A. Rogers, K. E. Paul, G. M. Whitesides, Chemical Reviews 1999, 99, 1823.

[16] H. W. C. Postma, T. Teepen, Z. Yao, M. Grifoni, C. Dekker, Science 2001, 293, 76.

[17] C. P. Collier, J. O. Jeppesen, Y. Luo, J. Perkins, E. W. Wong, J. R. Heath, et al., Journal of the American Chemical Society 2001, 123, 12632.

[18] R. F. Service, Science 2001, 294, 2442.

[19] J. E. Green, J. Wook Choi, A. Boukai, Y. Bunimovich, E. Johnston-Halperin, E. Delonno, et al., Nature 2007, 445, 414.

[20] S. E. Thompson, S. Parthasarathy, Materials Today 2006, 9, 20.

[21] F. Monnier, F. o. Turtaut, L. Duroure, M. Taillefer, Organic Letters 2008, 10, 3203.

[22] (a) L. Manchanda, M. D. Morris, M. L. Green, R. B. van Dover, F. Klemens, T. W. Sorsch, et al., Microelectronic Engineering 2001, 59, 351; (b) H. Wong, H. Iwai, Microelectronic Engineering 2006, 83, 1867; (c) R. L. McCreery, H. Yan, A. J. Bergren, Physical Chemistry Chemical Physics 2013, 15, 1065.

[23] J. Luo, M. Liu, Q. Zhao, J. Zhao, Y. Zhang, L. Tan, et al., Electrochimica Acta 2010, 56, 454.

[24] (a) B. A. Hylke, B. Bert de, Journal of Physics: Condensed Matter 2008, 20, 013001; (b) H. Haick, D. Cahen, Accounts of Chemical Research 2008, 41, 359.

[25] (a) R. L. McCreery, A. J. Bergren, Advanced Materials 2009, 21, 4303; (b) R. J. Nichols, W. Haiss, S. J. Higgins, E. Leary, S. Martin, D. Bethell, Physical Chemistry Chemical Physics 2010, $12,2801$.

[26] (a) M. Mayor, C. von Hänisch, H. B. Weber, J. Reichert, D. Beckmann, Angewandte Chemie International Edition 2002, 41, 1183; (b) K. Liu, X. Wang, F. Wang, ACS Nano 2008, 2, 2315.

[27] (a) S. Hong, R. Reifenberger, W. Tian, S. Datta, J. I. Henderson, C. P. Kubiak, Superlattices and Microstructures 2000, 28, 289; (b) S.-H. Ke, H. U. Baranger, W. Yang, Journal of the American Chemical Society 2004, 126, 15897; (c) F. Chen, X. Li, J. Hihath, Z. Huang, N. Tao, Journal of the American Chemical Society 2006, 128, 15874; (d) Z. L. Cheng, R. Skouta, V. H., J. R. Widawsky, S. Schneebeli, W. Chen, et al., Nat Nano 2011, 6, 353; (e) M. Frei, S. V. Aradhya, M. S. Hybertsen, L. Venkataraman, Journal of the American Chemical Society 2012, 
134, 4003; (f) T. A. Su, J. R. Widawsky, H. Li, R. S. Klausen, J. L. Leighton, M. L. Steigerwald, et al., Journal of the American Chemical Society 2013, 135, 18331.

[28] (a) W. Haiss, C. Wang, I. Grace, A. S. Batsanov, D. J. Schiffrin, S. J. Higgins, et al., Nat Mater 2006, 5, 995; (b) W. Haiss, C. Wang, R. Jitchati, I. Grace, S. Martín, A. S. Batsanov, et al., Journal of Physics: Condensed Matter 2008, 20, 374119.

[29] (a) Z. Li, B. Han, G. Meszaros, I. Pobelov, T. Wandlowski, A. Blaszczyk, et al., Faraday Discussions 2006, 131, 121; (b) J. R. Quinn, F. W. Foss, L. Venkataraman, R. Breslow, Journal of the American Chemical Society 2007, 129, 12376.

[30] J. G. Kushmerick, D. B. Holt, J. C. Yang, J. Naciri, M. H. Moore, R. Shashidhar, Physical Review Letters 2002, 89, 086802.

[31] S. Rigaut, Dalton Transactions 2013, 42, 15859.

[32] J. M. Tour, Molecular Electronics: Commercial Insights, Chemistry, Devices, Architecture and Programming 2003 (World Scientific Publishing: NJ, USA).

[33] P. F. H. Schwab, M. D. Levin, J. Michl, Chemical Reviews 1999, 99, 1863.

[34] K. Sonogashira. in Metal-Catalyzed Cross-Coupling Reactions 1st Ed 1998, Ch. 5, pp. 203-229 (Wiley-VCH: Weinheim, Germany).

[35] (a) M. A. Reed, J. Chen, A. M. Rawlett, D. W. Price, J. M. Tour, Applied Physics Letters 2001, 78, 3735; (b) F.-R. F. Fan, R. Y. Lai, J. Cornil, Y. Karzazi, J.-L. Brédas, L. Cai, et al., Journal of the American Chemical Society 2004, 126, 2568; (c) X. Xiao, L. A. Nagahara, A. M. Rawlett, N. Tao, Journal of the American Chemical Society 2005, 127, 9235; (d) A. S. Blum, J. G. Kushmerick, D. P. Long, C. H. Patterson, J. C. Yang, J. C. Henderson, et al., Nat Mater 2005, 4, 167; (e) Q. Lu, K. Liu, H. Zhang, Z. Du, X. Wang, F. Wang, ACS Nano 2009, 3, 3861.

[36] H. Hakkinen, Nat Chem 2012, 4, 443.

[37] (a) J. A. M. Sondag-Huethorst, C. Schonenberger, L. G. J. Fokkink, The Journal of Physical Chemistry 1994, 98, 6826; (b) G. Rubio, N. Agraït, S. Vieira, Physical Review Letters 1996, 76, 2302; (c) G. Rubio-Bollinger, S. R. Bahn, N. Agraït, K. W. Jacobsen, S. Vieira, Physical Review Letters 2001, 87, 026101; (d) D. Krüger, H. Fuchs, R. Rousseau, D. Marx, M. Parrinello, Physical Review Letters 2002, 89, 186402; (e) Huang, F. Chen, P. A. Bennett, Tao, Journal of the American Chemical Society 2007, 129, 13225.

[38] (a) Z. J. Donhauser, B. A. Mantooth, K. F. Kelly, L. A. Bumm, J. D. Monnell, J. J. Stapleton, et al., Science 2001, 292, 2303; (b) G. K. Ramachandran, T. J. Hopson, A. M. Rawlett, L. A. Nagahara, A. Primak, S. M. Lindsay, Science 2003, 300, 1413; (c) S. Yasuda, S. Yoshida, J. Sasaki, Y. Okutsu, T. Nakamura, A. Taninaka, et al., Journal of the American Chemical Society 2006, 128, 7746.

[39] (a) B. Xu, N. J. Tao, Science 2003, 301, 1221; (b) C. Wang, A. S. Batsanov, M. R. Bryce, S. Martín, R. J. Nichols, S. J. Higgins, et al., Journal of the American Chemical Society 2009, 131, 15647; (c) Y. le, T. Hirose, H. Nakamura, M. Kiguchi, N. Takagi, M. Kawai, et al., Journal of the American Chemical Society 2011, 133, 3014; (d) G. Sedghi, V. M. Garcia-Suarez, L. J. Esdaile, H. L. Anderson, C. J. Lambert, S. Martin, et al., Nat Nano 2011, 6, 517; (e) X. Zhao, C. Huang, M. Gulcur, A. S. Batsanov, M. Baghernejad, W. Hong, et al., Chemistry of Materials 2013, 25, 4340; (f) L. Venkataraman, J. E. Klare, I. W. Tam, C. Nuckolls, M. S. Hybertsen, M. L. Steigerwald, Nano Letters 2006, 6, 458; (g) L. Venkataraman, Y. S. Park, A. C. Whalley, C. Nuckolls, M. S. Hybertsen, M. L. Steigerwald, Nano Letters 2007, 7, 502; (h) L. Patrone, S. Palacin, J. Charlier, F. Armand, J. P. Bourgoin, H. Tang, et al., Physical Review Letters 2003, 91, 096802; (i) P. MorenoGarcía, M. Gulcur, D. Z. Manrique, T. Pope, W. Hong, V. Kaliginedi, et al., Journal of the American Chemical Society 2013, 135, 12228; (j) S. Martin, W. Haiss, S. J. Higgins, R. J. Nichols, Nano Letters 2010, 10, 2019; (k) M. Kiguchi, S. Miura, K. Hara, M. Sawamura, K. Murakoshi, Applied Physics Letters 2006, 89, 213104; (I) J. Chen, L. C. Calvet, M. A. Reed, D. W. Carr, D. S. Grubisha, D. W. Bennett, Chemical Physics Letters 1999, 313, 741; (m) J. M. Beebe, V. B. Engelkes, L. L. Miller, C. D. Frisbie, Journal of the American Chemical Society 2002, 124, 11268; (n) M.-D. Fu, I. W. P. Chen, H.-C. Lu, C.-T. Kuo, W.-H. Tseng, C.-h. Chen, The Journal of Physical Chemistry C 2007, 111, 11450; (o) A. Fukazawa, M. Kiguchi, S. Tange, Y. Ichihashi, Q. Zhao, T. 
Takahashi, et al., Chemistry Letters 2011, 40, 174; (p) G. Pera, S. Martín, L. M. Ballesteros, A. J. Hope, P. J. Low, R. J. Nichols, et al., Chemistry - A European Journal 2010, 16, 13398; (q) S. Marques-Gonzalez, D. S. Yufit, J. A. K. Howard, S. Martin, H. M. Osorio, V. M. Garcia-Suarez, et al., Dalton Transactions 2013, 42, 338; (r) Y. Komoto, S. Fujii, K. Hara, M. Kiguchi, The Journal of Physical Chemistry C 2013, 117, 24277; (s) W. Hong, H. Li, S.-X. Liu, Y. Fu, J. Li, V. Kaliginedi, et al., Journal of the American Chemical Society 2012, 134, 19425.

[40] M. J. Huang, L. Y. Hsu, M. D. Fu, S. T. Chuang, F. W. Tien, C. H. Chen, Journal of the American Chemical Society 2014, 136, 1832.

[41] X. Crispin, V. Geskin, A. Crispin, J. Cornil, R. Lazzaroni, W. R. Salaneck, et al., Journal of the American Chemical Society 2002, 124, 8131.

[42] N. J. Tao, Physical Review Letters 1996, 76,

[43] G. Wang, T. W. Kim, T. Lee, W. Wang, M. A. Reed. in Comprehensive Nanoscience and Technology (Andrews DL, Scholes GD, Wiederrecht GP) 2011, Ch. 4.16, pp. 463-487 (Academic Press: Amsterdam).

[44] (a) A. Nitzan, Annual Review of Physical Chemistry 2001, 52, 681; (b) A. Nitzan, M. A. Ratner, Science 2003, 300, 1384; (c) L. Malysheva, A. Onipko, physica status solidi (b) 2007, 244, 4244; (d) N. A. Zimbovskaya, M. R. Pederson, Physics Reports 2011, 509, 1.

[45] (a) D. M. Adams, L. Brus, C. E. D. Chidsey, S. Creager, C. Creutz, C. R. Kagan, et al., The Journal of Physical Chemistry B 2003, 107, 6668. (b) C. Chiorboli, M.T. Indelli, F. Scandola, Top. Curr. Chem. 2005, 257, 63.

[46] R. Yamada, H. Kumazawa, T. Noutoshi, S. Tanaka, H. Tada, Nano Letters 2008, 8, 1237.

[47] V. Kaliginedi, P. Moreno-García, H. Valkenier, W. Hong, V. M. García-Suárez, P. Buiter, et al., Journal of the American Chemical Society 2012, 134, 5262.

[48] (a) D. J. Wold, R. Haag, M. A. Rampi, C. D. Frisbie, The Journal of Physical Chemistry $B$ 2002, 106, 2813; (b) T. Ishida, W. Mizutani, Y. Aya, H. Ogiso, S. Sasaki, H. Tokumoto, The Journal of Physical Chemistry B 2002, 106, 5886.

[49] (a) H. Liu, N. Wang, J. Zhao, Y. Guo, X. Yin, F. Y. C. Boey, et al., ChemPhysChem 2008, 9, 1416; (b) M. D. Newton, J. F. Smalley, Physical Chemistry Chemical Physics 2007, 9, 555.

[50] (a) J. N. Onuchic, D. N. Beratan, J. R. Winkler, H. B. Gray, Annual Review of Biophysics and Biomolecular Structure 1992, 21, 349; (b) O. S. Wenger, Accounts of Chemical Research 2010, 44, 25.

[51] C. Joachim, M. A. Ratner, Nanotechnology 2004, 15, 1065.

[52] W. B. Davis, W. A. Svec, M. A. Ratner, M. R. Wasielewski, Nature 1998, 396, 60.

[53] S. Ho Choi, B. Kim, C. D. Frisbie, Science 2008, 320, 1482.

[54] (a) L. Luo, S. H. Choi, C. D. Frisbie, Chemistry of Materials 2010, 23, 631; (b) E. Wierzbinski, R. Venkatramani, K. L. Davis, S. Bezer, J. Kong, Y. Xing, et al., ACS Nano 2013, 7, 5391; (c) N. Renaud, Y. A. Berlin, F. D. Lewis, M. A. Ratner, Journal of the American Chemical Society 2013, 135, 3953.

[55] (a) A. Nitzan, The Journal of Physical Chemistry A 2001, 105, 2677; (b) Y. A. Berlin, M. A. Ratner, Radiation Physics and Chemistry 2005, 74, 124; (c) M. C. Traub, B. S. Brunschwig, N. S. Lewis, The Journal of Physical Chemistry B 2007, 111, 6676.

[56] J. P. Bergfield, M. A. Ratner, physica status solidi (b) 2013, 250, 2249.

[57] A. Nitzan, Israel Journal of Chemistry 2002, 42, 163.

[58] J. R. Heath, M. A. Ratner, Physics Today 2003, 56, 43. 